\title{
Mediastinal/hilar lymphadenopathy: A possibly overlooked variable in the classification of lung nodules
}

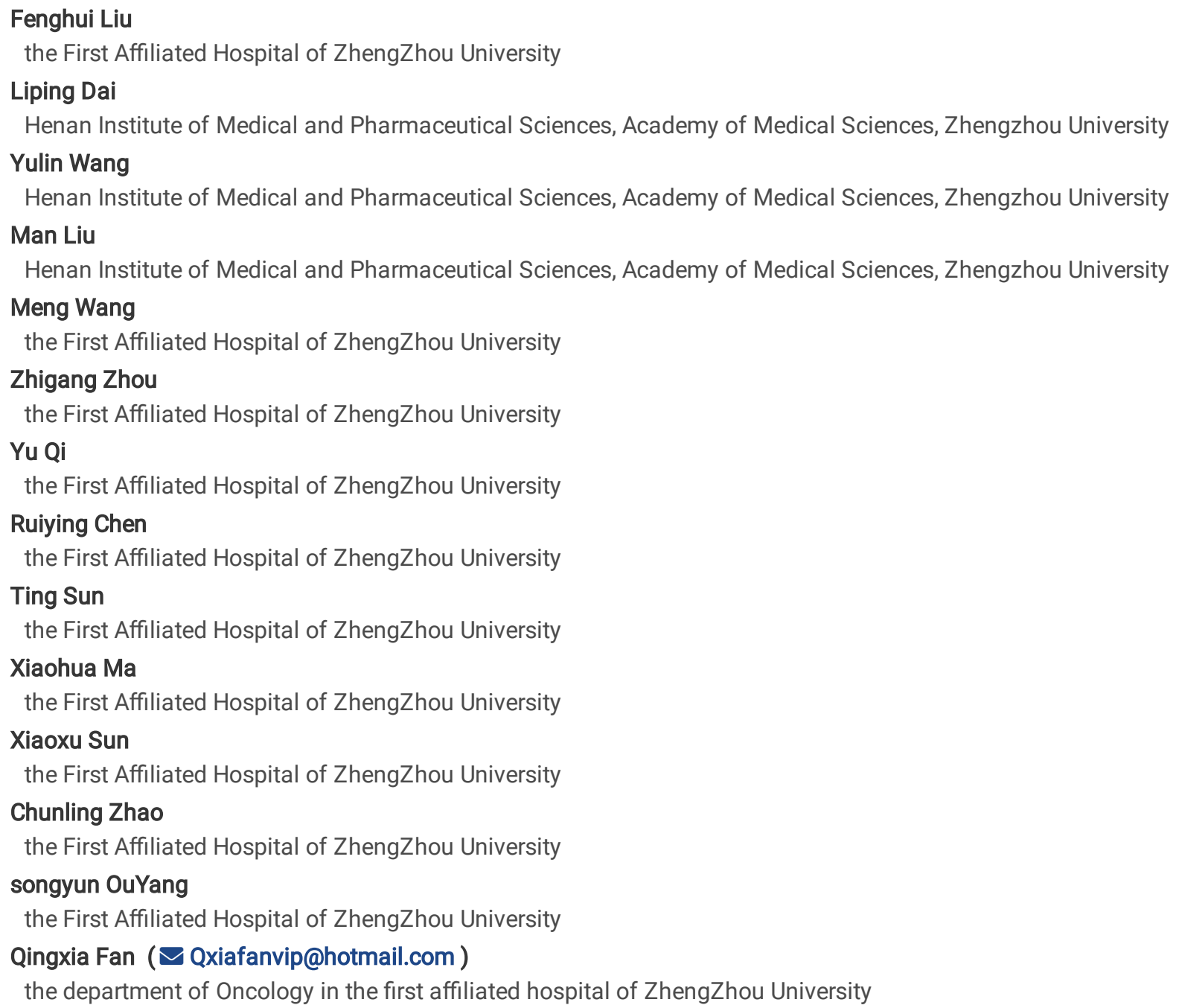

Research article

Keywords: Lung nodule, Mediastinal/hilar lymphadenopathy, Prediction Model, Nomogram

Posted Date: November 1st, 2021

DOI: https://doi.org/10.21203/rs.3.rs-958972/v1

License: (c) (i) This work is licensed under a Creative Commons Attribution 4.0 International License. Read Full License 


\section{Abstract \\ Background}

Clinical prediction models to classify lung nodules often exclude patients with mediastinal/hilar lymphadenopathy, although the presence of mediastinal/hilar lymphadenopathy does not always indicate malignancy. Herein, we developed and validated a multimodal prediction model for lung nodules in which patients with mediastinal/hilar lymphadenopathy were included.

\section{Methods}

A total of 359 patients with pulmonary nodules were considered for enrollment in the study. We developed and validated a logistic regression model including patients with mediastinal/hilar lymphadenopathy. Discrimination of the model was assessed by area under the operating curve. Goodness of fit was performed via the Hosmer-Lemeshow test, and a nomogram of the logistic regression model was drawn.

\section{Results}

There were 311 cases included in the final analysis. A logistic regression model was developed and validated. There were nine independent variables included in the model. The AUC of the training and validation sets was $0.93(95 \% \mathrm{Cl}, 0.90-0.97)$ and $0.91(95 \% \mathrm{Cl}, 0.85-0.98)$, respectively. In the validation set with or without mediastinal/hilar lymphadenopathy, the AUC was $0.95(95 \% \mathrm{Cl}, 0.90-0.99)$ and 0.91 $(95 \% \mathrm{Cl}, 0.87-0.95)$, respectively. The Hosmer-Lemeshow goodness-of-fit statistic was 0.22 . A nomogram was drawn to visualize the model.

\section{Conclusions}

We developed and validated a multimodal risk prediction model for lung nodules with excellent discrimination and calibration, regardless of the inclusion of mediastinal/hilar lymphadenopathy. This broadens the application of lung nodule prediction models. Furthermore, the presence of mediastinal/hilar lymphadenopathy added value for predicting lung nodule malignancy, highlighting the importance of this variable in clinical practice.

\section{Introduction}

Lung cancer is the leading cause of cancer related deaths worldwide (1), particularly in China (2). It is estimated (3) that in 2018, there were

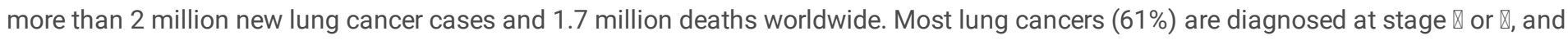
only $21 \%$ of cases are diagnosed at stage $\Downarrow$. The 5 -year survival rate of patients with NSCLC is $57 \%$ for stage $\rrbracket$, while it declines to $4 \%$ for stage $\otimes(4)$. Early detection of lung cancer can increase the cure rate of lung cancer and prolong the survival time of lung cancer patients.

With the wide application of low-dose computed tomography (LDCT), early screening for lung cancer is now available. The NSLT study (5) suggested that compared with chest X-rays, screening in high-risk populations with LDCT could reduce the death rate from lung cancer by $20 \%$. However, the study also showed that the false positive rate for screening was as high as $96 \%$. False positive and unclear results may lead to additional, unnecessary examinations and increase mental stress in patients. Bach PB et al. (6) reported that CT screening led to a three-fold increase in the rate of diagnosis of lung cancer, while the number of lung cancer-related surgeries increased by 10 times. The identification of lung nodules is a great mental burden for participants and their families. Repeated follow-up and invasive examinations not only increase the social and economic burden on patients, but also interfere with their normal work and life.

The classic definition of a solitary pulmonary nodule (SPN) is a circular nodule less than or equal to $30 \mathrm{~mm}$ in diameter surrounded by normal lung tissue that is not accompanied by atelectasis, lymph node enlargement, or pleural effusion (7). Many prediction models of SPN have been developed and validated, such as the Mayo model (8), VA model (9), Brock model (10), and PKUPH model (11), which have achieved excellent performance in discrimination and calibration. The variables included in the models include sociodemographic factors, smoking, CT characteristics, and tumor biomarkers. The AUCs of the models ranged from 0.79 to 0.91 . Some of the models were validated with external datasets and achieved outstanding discrimination and calibration (12).

However, pulmonary nodules of unknown nature are not only limited to SPN in clinical practice. Multiple pulmonary nodules and lung nodules with mediastinal/hilar lymphadenopathy are also common clinical challenges, and the definition of a pulmonary nodule should

Page 2/13 
therefore be updated (13), (14). Mediastinal/hilar lymph nodes have a short axis diameter $>10 \mathrm{~mm}$, while subcarinal nodes have a short axis $>12 \mathrm{~mm}$; this is defined as mediastinal/hilar lymphadenopathy (15). Mediastinal/hilar lymphadenopathy may be benign, malignant, or reactive (16), and can be seen in malignant diseases such as tumor metastasis and lymphoma as well as benign diseases such as nonspecific infection, interstitial lung disease, tuberculosis, and sarcoidosis $(17,18)$. The definition of a solitary pulmonary nodule currently excludes mediastinal/hilar lymphadenopathy, which limits the application of previous clinical prediction models of pulmonary nodules.

The efficient identification of high-risk lung nodules and the reduction of unnecessary follow-up or invasive examinations for benign nodules are urgent clinical issues and of interest to researchers. No model currently exists for use in current clinical practice. The purpose of this study was to develop and validate a multimodal risk prediction model of pulmonary nodules that includes mediastinal/hilar lymphadenopathy, and to explore whether this is of value to the prediction of lung nodule malignancy. The results may be used to broaden the scope of clinical application of this prediction model for lung nodules.

\section{Patients And Methods}

\section{Patients}

Patients with lung nodules detected by chest CT, who were admitted to the Department of Respiration, Thoracic Surgery, Imaging and Nuclear Medicine of the First Affiliated Hospital of Zhengzhou University (ZZU) between November 2016 and March 2019, were included. All the participants in this study came from central China. The study was approved by the Ethics Committee of the First Affiliated Hospital of Zhengzhou University, and all patients gave written consent. Patients who required further invasive procedures, such as CT-guided lung biopsy, bronchoscopy, or lung surgery signed the corresponding informed consent.

\section{Inclusion criteria}

(1) Age > 16 years; (2) lung nodules with a major axis (2-30 mm); (3) had a definite diagnosis.

\section{Exclusion criteria}

(1) Concomitant with pleural effusion, direct mediastinal invasion, or distant metastasis; (2) chest imaging follow-up time was less than two years or was difficult to identify, or patient was lost to follow-up; (3) lung nodules secondary to other solid tumors; and (4) chest radiographic data were missing or untraceable.

The study was designed and conducted in accordance with the Transparent Reporting of a Multivariate Prediction Model for Individual Prognosis or Development (TRIPOD) statement (19).

\section{Variables}

This study included the following variables: demographic information (age, gender, date of admission), traditional risk factors (smoking history, drinking history, family history of cancer), chest CT features of the nodule (shape, type, size [major axis], number, margin, cavity, burr sign, vascular notch, lobulation, crest, pleural traction, mediastinal/hilar lymphadenopathy, emphysema, calcification), and traditional tumor markers including carcinoembryonic antigen (CEA), carbohydrate antigen 125 (CA125), CYFRA211, and neuron-specific enolase (NSE), which were obtained from the electronic medical database of our hospital. Based on study design, $70 \%$ of the included patients were randomly selected as the training set, and the remaining patients were the validation set.

CT scans were performed using a 64-slice Lightspeed spiral CT from Philips Brilliance of the Netherlands or GE of the United States. The tube voltage was set at $120 \mathrm{kVP}$, the tube current was $40 \mathrm{~mA}$, and the layer thickness was $0.6-1.5 \mathrm{~mm}$ by thin layer reconstruction. The analysis window was lung window (window width $1200 \mathrm{U}$, window position $-600 \mathrm{U}$ ). The $\mathrm{CT}$ features of the lung nodules were independently determined by two senior attending physicians from the Department of Respiratory Medicine (SY Ouyang) and the Department of radiology ( $M$ Wang), and disagreements were discussed and resolved with a third doctor (ZG Zhou).

\section{Statistical analysis}

The missing data were replaced using linear interpolation. Variables were presented as number (percent), mean (SD), or median (interquartile [IQR]), as appropriate. To compare benign and malignant groups, we used independent sample $t$ tests for continuous variables and the chi-square test for categorized variables. Univariate analysis was performed to identify potential risk factors. Multivariate 
logistic regression was used to establish, evaluate, optimize, and validate the clinical prediction model. The AUC, sensitivity, specificity, positive predictive value (PPV), negative predictive value (NPV), and accuracy of the model were then calculated. AUC and HosmerLemeshow fitting tests were employed to assess the discrimination and calibration of the model, respectively. The risk prediction model for malignancy of pulmonary nodules (ZZU model) was then established and validated. Subgroup validation was performed according to the presence or absence of mediastinal/hilar lymphadenopathy. A nomogram of the logistic regression model was then drawn. A two-tailed $p<$ 0.05 was considered statistically significant. All statistical analyses were conducted using SPSS version 21.0 (IBM Corporation, Chicago) and $\mathrm{R}$ version 3.6.1 (R Foundation for Statistical Computing). Figure 1 provides a flowchart of the study.

\section{Results}

\section{Patient characteristics}

Of the 359 participants with lung nodules who were considered for the study, 48 were excluded from the final analysis ( 22 had no CT imaging or tumor biomarkers or were lost to follow-up, 21 had no definite diagnosis, 5 had lung metastasis). Finally, 311 patients with a definite diagnosis were included in our analysis, and the median follow-up time was 18 months. Of these patients, 129 were categorized as having malignant nodules, 173 were male, and the mean age was 57 years old. The pathology of the lung nodules is shown in Table 1; nine participants in the benign group had a clinical diagnosis.

Table 1

The pathology of lung nodules

\begin{tabular}{|c|c|c|c|}
\hline \multicolumn{2}{|c|}{ Pathology of lung nodule } & \multirow{2}{*}{$\begin{array}{l}\text { Frequency } \\
29\end{array}$} & \multirow{2}{*}{$\begin{array}{l}\text { Percent (\%) } \\
22.5\end{array}$} \\
\hline Benign & Tuberculosis & & \\
\hline & pneumomycosis & 9 & 7.1 \\
\hline & Chronic inflammation & 51 & 39.5 \\
\hline & Organized pneumonia & 2 & 1.6 \\
\hline & hamartoma & 7 & 5.4 \\
\hline & pulmonary sclerosing pneumocytoma & 1 & 0.8 \\
\hline & granuloma & 6 & 4.7 \\
\hline & Other inflammation & 15 & 11.6 \\
\hline & Total & 129 & 100 \\
\hline \multirow[t]{7}{*}{ Malignant } & squamous carcinoma & 22 & 12.1 \\
\hline & adenocarcinoma & 130 & 71.3 \\
\hline & Small cell lung cancer & 12 & 6.6 \\
\hline & Large cell carcinoma & 1 & .5 \\
\hline & Adenoid cystic carcinoma & 5 & 2.7 \\
\hline & Undifferentiated carcinoma & 12 & 6.6 \\
\hline & Total & 182 & 100.0 \\
\hline
\end{tabular}

\section{Univariate and multivariate logistic regression analysis}

As shown in Table 2, age, smoking history, and family history of cancer, nodule number, size, shape, margin, burr sign, vascular notch, lobulation sign, crest, pleural traction sign, mediastinal/hilar lymphadenopathy, calcification, CEA, CA125, and CYFRA211 were statistically different between the benign and malignant groups, based on univariate analysis. Except for lung nodule diameter, there were no significant differences in clinical features between the training and validation sets. The mean size was $18.49 \pm 7.37$ in the training set and $16.42 \pm 8.70$ in the validation set, which is a minimal difference in clinical practice. 
Table 2

Comparison of clinical features between benign and malignant groups in the derivation set

\begin{tabular}{|c|c|c|c|c|c|}
\hline Variable & Total & benign (\%) & malignant (\%) & Wald X2 & $\mathrm{p}$ \\
\hline Gender & 212 & 92 & 120 & 1.349 & 0.291 \\
\hline male & 125 & $58(63.0)$ & $67(55.8)$ & & \\
\hline female & 87 & $34(37.0)$ & $53(44.2)$ & & \\
\hline Age & 212 & $51.83 \pm 13.10$ & $61.42 \pm 10.56$ & 25.160 & 0.000 \\
\hline Smoking & 67 & $20(21.7)$ & 47 (39.2) & 7.148 & 0.008 \\
\hline Drinking & 36 & $12(14.3)$ & $24(20.7)$ & 1.354 & 0.245 \\
\hline Family history of cancer & 14 & $3(3.3)$ & $11(9.2)$ & 2.704 & 0.100 \\
\hline Number & 212 & 92 & 120 & 13.282 & 0.000 \\
\hline 1 & 144 & $50(54.3)$ & $94(78.3)$ & & \\
\hline$\geq 2$ & 68 & $42(45.7)$ & $26(21.7)$ & & \\
\hline Type & 212 & 92 & 120 & 0.734 & 0.392 \\
\hline solid & 152 & $69(75.0)$ & $83(69.2)$ & & \\
\hline GGO & 34 & $13(14.1)$ & $21(17.5)$ & & \\
\hline subsolid & 26 & $10(10.9)$ & $16(13.3)$ & & \\
\hline Size & 212 & $16.46 \pm 7.77$ & $20.04 \pm 6.66$ & 11.832 & 0.001 \\
\hline Shape & 212 & 92 & 120 & 9.790 & 0.002 \\
\hline circle & 58 & $33(35.9)$ & $25(20.8)$ & & \\
\hline oval & 49 & $25(27.2)$ & $24(23.1)$ & & \\
\hline unregular & 105 & $34(37.0)$ & 71 (59.2) & & \\
\hline Margin & 212 & 92 & 120 & 1.220 & 0.269 \\
\hline clear & 134 & $62(67.4)$ & $72(60.0)$ & & \\
\hline ambiguous & 78 & $30(32.6)$ & $48(36.8)$ & & \\
\hline Cavity & 28 & $11(12.0)$ & $17(14.2)$ & 0.221 & 0.638 \\
\hline Vascular notch & 147 & $42(45.7)$ & $105(87.5)$ & 37.463 & 0.000 \\
\hline Lobulation & 79 & $9(9.8)$ & $70(58.3)$ & 41.563 & 0.000 \\
\hline Burr sign & 73 & $13(14.1)$ & $60(50.0)$ & 26.492 & 0.000 \\
\hline Pleural traction & 46 & $12(13.0)$ & $34(28.3)$ & 6.862 & 0.009 \\
\hline Crest & 38 & $4(4.3)$ & $34(28.3)$ & 15.472 & 0.000 \\
\hline Mediastinal/hilar lymphadenopathy & 42 & $12(13.0)$ & 40 (33.3) & 10.872 & 0.001 \\
\hline Emphysema & 49 & $17(18.5)$ & $32(26.7)$ & 1.947 & 0.163 \\
\hline Calcification & 21 & $16(17.4)$ & $5(4.2)$ & 8.750 & 0.003 \\
\hline CEA & 212 & $1.99 \pm 1.71$ & $69.55 \pm 6.38$ & 11.185 & 0.001 \\
\hline CA125 & 206 & $14.16 \pm 11.72$ & $38.87 \pm 43.70$ & 8.780 & 0.003 \\
\hline CYFRA211 & 206 & $2.01 \pm 1.15$ & $4.67 \pm 12.33$ & 13.426 & 0.000 \\
\hline NSE & 205 & $12.74 \pm 5.77$ & $12.21 \pm 8.61$ & 0.258 & 0.611 \\
\hline
\end{tabular}


In the univariate regression analysis, risk factors associated with lung nodule malignancy included older age, smoking, fewer nodules, longer diameter, nonregular shape, vascular notch, lobulation, burr sign, pleural traction, crest, mediastinal/hilar lymphadenopathy, no calcification, higher CEA value, CA125, and CYFRA211. In the multivariate logistic regression analysis, number, vascular notch, mediastinal/hilar lymphadenopathy, lobulation, age, gender, CEA, CA125, and CYFRA211 were associated with lung nodule malignancy (Table 3).

Table 3

The univariate and multivariate analysis of risk factors for lung nodule malignancy

\begin{tabular}{|lllllll|}
\hline Variable & Univariate analysis & \multicolumn{4}{l|}{ Multivariate analysis } \\
\hline & OR & $95 \% \mathrm{Cl}$ & $\mathrm{p}$ & $\mathrm{OR}$ & $95 \% \mathrm{Cl}$ & $\mathrm{p}$ \\
\hline Number & 0.329 & $0.181-0.598$ & 0.000 & .163 & $0.058-0.453$ & 0.001 \\
\hline Vascular notch & 8.333 & $4.226-16.432$ & 0.000 & 4.641 & $1.814-11.874$ & 0.001 \\
\hline Lobulation & 12.911 & $5.932-28.101$ & 0.000 & 10.921 & $4.024-29.636$ & 0.000 \\
\hline ML & 3.333 & $1.630-6.819$ & 0.001 & 2.974 & $1.050-8.425$ & 0.040 \\
\hline Age & 1.073 & $1.044-1.103$ & 0.000 & 1.073 & $1.025-1.124$ & 0.003 \\
\hline Gender & 1.349 & $0.774-2.353$ & 0.291 & 3.277 & $1.305-8.228$ & 0.011 \\
\hline CEA & 1.309 & $1.118-1.533$ & 0.001 & 1.151 & $0.939-1.410$ & 0.176 \\
\hline CA125 & 1.028 & $1.009-1.046$ & 0.003 & 1.039 & $1.011-1.068$ & 0.006 \\
\hline CYFRA211 & 1.432 & $1.182-1.736$ & 0.000 & 1.344 & $0.994-1.817$ & 0.055 \\
\hline Abbreviations: OR: odds ratio, Cl: confidence interval, ML: Mediastinal/hilar lymphadenopathy. \\
\hline
\end{tabular}

\section{The ZZU model and ROC curves}

Regression equation of the ZZU prediction model: $\mathrm{X}=$

-7.619-1.187*number+1.535*vascular+2.391*lobulation+1.090*ML+0.07*age+0.140*CEA+0.038*CA125+0.295*CYFRA211+1.187*gender

\section{$P=\exp (X) /(1+\exp (X))$}

The performance of the regression equation was assessed by ROC curve analysis, as shown in Figure 2 . The AUC of the ZZU model in the derivation cohort was $0.93(95 \% \mathrm{Cl} 0.90-0.97)$ and the AUC of the ZZU model in the validation set was 0.91 (95\% $\mathrm{Cl} 0.85-0.98)$. Validation of lung nodules with mediastinal/hilar lymphadenopathy: the AUC of the model was 0.95 (95\% Cl: 0.90-0.99). Validation of lung nodules without mediastinal/hilar lymphadenopathy: the AUC of the model was 0.91 (95\% Cl: 0.87-0.95).

The cut-off value was calculated by Youden index, and the maximum of the Youden index was 0.734 . The predicted cut-off value was 0.6279 , the sensitivity was $83.2 \%$, the specificity was $90.2 \%$, the positive predictive value (PPV) was $88.2 \%$, the negative predictive value was $83.7 \%$, and the accuracy was $86.3 \%$. The Hosmer-Lemeshow goodness-of-fit test result was 0.221 ( $p>0.05$ ). The expected value and observed values fit well, as shown in Figure 3.

\section{Nomogram of clinical prediction model for lung nodules}

The nomogram of the prediction model for malignancy of lung nodules is shown in Figure 4, and eight independent variables were selected and visualized. We calculated the malignant risk of lung nodules based on the variables.

\section{Discussion}

We developed and validated a multimodal risk prediction model for solitary and multiple pulmonary nodules. Based on clinical practice, mediastinal/hilar lymph node enlargement was included in the study and identified as an independent variable. Stemming from a realworld clinical background, this study enrolled patients with pulmonary nodules identified due to health examination or respiratory symptoms, to more objectively reflect the actual needs of clinical practice. 
The rate of smoking was $21.7 \%$ and $39.2 \%$ in the benign and malignant groups, respectively, which is lower than in previous lung cancer screening programs. Most of female participants in our study had no history of smoking, and smoking was not identified as an independent variable. Female sex was an indicator for lung nodule malignancy, the OR of which was 3.277 (95\% $\mathrm{Cl}, 1.305-8.228$ ). Another study identified female sex as an important predictor of lung cancer in the highly prevalent non-smoking related lung cancers in Taiwan (20). During 2002 and 2012, Korean women without a history of smoking had an increasing trend of lung cancer morbidity(21). In northern China, the epidemiological risk factors for lung cancer in never-smokers included exposure to passive smoking, cooking habits, disease history, and a family history of lung cancer (22).

Mediastinal/hilar lymphadenopathy does not always indicate malignancy. In this study, the positive rate was 10.9\% (14/129) in the benign group and 32.4\% (59/182) in the malignant group. The differential diagnosis of mediastinal/hilar lymphadenopathy is difficult, and ultrasound elastography, endobronchial ultrasound transbronchial needle aspiration (EBUS-TBNA), or surgery may be needed to accomplish this task (17), (23), (24). The classic definition of lung nodules is not suitable for current clinical practice. Univariate analysis indicated that mediastinal/hilar lymphadenopathy was statistically different between the malignant and benign groups. Subsequent multivariate logistic regression analysis revealed it to be an independent predictor.

Using a risk prediction model is superior to the present lung cancer screening guidelines, although discrimination ranges widely (AUCs from 0.61 to 0.81 ) (25-27). Owing to differences in heredity, smoking status, and region and industrialization, foreign models cannot be always applied to the Chinese population. A recent study based on patients from northwest China validated the Mayo model, VA model, and PKUPH model, although the AUCs were only $0.655,0.603$, and 0.521 , respectively (28).

We developed a ZZU model suitable for classification of pulmonary nodules regardless of the presence of mediastinal/hilar lymphadenopathy. The variables included in the equation were sorted in order of importance: lobulation, vascular notch, gender, mediastinal/hilar lymphadenopathy, CYFRA211, CEA, age, CA125, and number of nodules. The AUCs of the training set and the validation set were 0.931 and 0.913 , respectively, and the Hosmer-Lemeshow fit test $(p>0.05)$ suggested excellent calibration. This was the highest among the current lung cancer risk prediction models for lung nodules(29), (30). Both subgroups, regardless of the presence of mediastinal/hilar lymphadenopathy, achieved high discrimination, while the predictive ability of the subgroup with mediastinal/hilar lymphadenopathy was superior.

Strengths of our research: Compared with a variety of lung nodule risk prediction models, we have developed and validated a clinical prediction model suitable for multiple lung nodules that broadens the prediction range of lung nodules. Mediastinal/hilar lymphadenopathy was identified as an independent variable and was proved to have value in predicting lung nodule malignancy. The included variables are commonly used clinical indicators, which are more suitable for clinical applications and are easy to promote in areas with limited clinical resources. The ZZU model achieved excellent discrimination and calibration.

However, our study had some limitations. Our study was a single-center clinical study. Some cases had missing data, and some cases lacked a definitive pathological diagnosis and relied on a clinical diagnosis. For our lung nodule prediction model, sample size was a limiting factor for prediction accuracy. In addition, the tumor markers included are commonly used indicators for lung cancer. Although they are convenient and easy to obtain, they may not be the most suitable predictors for malignant lung nodules. With in-depth research and the further development of imaging and molecular biology, the selection of variables and the optimization of prediction algorithms will require regular updating.

\section{Conclusions}

Using a dataset derived from central China, we developed a ZZU model for lung nodules. The model achieved excellent discrimination and calibration. Mediastinal/hilar lymphadenopathy was included in the prediction model for the first time, and was identified as an independent variable in the model. We have broadened the clinical application of prediction models for predicting lung nodule malignancy. The ZZU model could be implemented to assist clinicians and patients with lung nodules regardless of the presence of mediastinal/hilar lymphadenopathy.

\section{Abbreviations}

AUC (area under the operating curve), ROC (receiver operating curve), ZZU (ZhengZhou University), NSCLC (non-small cell lung cancer), LDCT (low-dose computed tomography), SPN (solitude pulmonary nodule), CEA (carcinoembryonic antigen), CA125 (carbohydrate antigen 125), CYFRA211 (cytokeratin 19 fragment), NSE (neuron-specific enolase), PPV (positive predictive value), NPV (negative predictive value), SD (standard deviation), OR (odds ratio), Cl (confidence interval), EBUS-TBNA (endobronchial ultrasound transbronchial needle aspiration), 
ML (mediastinal/hilar lymphadenopathy), TRIPOD (Transparent Reporting of a Multivariate Prediction Model for Individual Prognosis or Development).

\section{Declarations}

\section{Ethics approval and consent to participate}

The study was approved by the Ethics Committee of the First Affiliated Hospital of Zhengzhou University, and all patients gave written consent.

\section{Consent for publication}

The final submission of the version and publication was approved by all of the authors.

\section{Availability of data and materials}

The datasets during and/or analyzed during the current study available from the corresponding author on reasonable request.

\section{Competing interests}

All authors have no conflicts of interest to declare.

\section{Funding}

This work was supported by the National Natural Science Foundation of China (Grant Number 8167291), the Leading Talents of Science and Technology Innovation in Henan Province (Grant Number 20420051008), and National Natural Science Foundation of China-Henan joint fund (Grant Number U1804195).

\section{Authors' contributions}

Fenghui Liu: Conceptualization, Data curation, Formal analysis, Investigation, Methodology, Software, Visualization, Writing - original draft.

Liping Dai: conceptualization, Formal analysis, Funding acquisition, Methodology, Software, Writing - original draft.

Yulin Wang: Data curation, Formal analysis, Investigation.

Man Liu: Data curation, Investigation.

Meng Wang: Data curation, Investigation.

Zhigang Zhou: Resources, Validation.

Yu Qi: Resources, Validation.

Ruiying Chen: Resources, Validation.

Ting Sun: Data curation, Investigation.

Xiaoxu Sun: Methodology, Resources, Software.

Xiaohua Ma: Data curation, Investigation.

Chunling Zhao: Data curation.

Songyun OuYang: conceptualization, Funding acquisition, Project administration, Resources, Supervision, Writing - review \& editing.

Qingxia Fan: conceptualization, Project administration, Resources, Validation, Supervision, Writing - review \& editing.

All authors read and approved the final manuscript. 
The authors thank Dr. Gao Yonghua for his help in proofreading and polishing the manuscript. We thank LetPub (www.letpub.com) for its linguistic assistance during the preparation of this manuscript.

\section{References}

1. Torre LA, Siegel RL, Jemal A. Lung Cancer Statistics. Adv Exp Med Biol. 2016;893:1-19.

2. Zheng RS, Sun KX, Zhang SW, Zeng HM, Zou XN, Chen R, et al. [Report of cancer epidemiology in China, 2015]. Zhonghua Zhong Liu Za Zhi. 2019;41(1):19-28.

3. Miranda-Filho A, Pineros M, Bray F. The descriptive epidemiology of lung cancer and tobacco control: a global overview 2018. Salud Publica Mex. 2019;61(3):219-29.

4. Miller KD, Nogueira L, Mariotto AB, Rowland JH, Yabroff KR, Alfano CM, et al. Cancer treatment and survivorship statistics, 2019. Cancer J Clin. 2019;69(5):363-85.

5. Kramer BS, Berg CD, Aberle DR, Prorok PC. Lung cancer screening with low-dose helical CT: results from the National Lung Screening Trial (NLST). J Med Screen. 2011;18(3):109-11.

6. Bach PB, Kattan MW, Thornquist MD, Kris MG, Tate RC, Barnett MJ, et al. Variations in lung cancer risk among smokers. J Natl Cancer Inst. 2003;95(6):470-8.

7. Gould MK, Donington J, Lynch WR, Mazzone PJ, Midthun DE, Naidich DP, et al. Evaluation of individuals with pulmonary nodules: when is it lung cancer? Diagnosis and management of lung cancer, 3rd ed: American College of Chest Physicians evidence-based clinical practice guidelines. Chest. 2013;143(5 Suppl):e93S-120S.

8. Swensen SJ, Silverstein MD, Ilstrup DM, Schleck CD, Edell ES. The probability of malignancy in solitary pulmonary nodules. Application to small radiologically indeterminate nodules. Arch Intern Med. 1997;157(8):849-55.

9. Gould MK, Ananth L, Barnett PG, Veterans Affairs SCSG. A clinical model to estimate the pretest probability of lung cancer in patients with solitary pulmonary nodules. Chest. 2007;131(2):383-8.

10. Tammemagi MC, Freedman MT, Pinsky PF, Oken MM, Hu P, Riley TL, et al. Prediction of true positive lung cancers in individuals with abnormal suspicious chest radiographs: a prostate, lung, colorectal, and ovarian cancer screening trial study. J Thorac Oncol. 2009;4(6):710-21.

11. Xiao F, Liu D, Guo Y, Shi B, Song Z, Tian Y, et al. Novel and convenient method to evaluate the character of solitary pulmonary nodulecomparison of three mathematical prediction models and further stratification of risk factors. PLoS One. 2013;8(10):e78271.

12. Winter A, Aberle DR, Hsu W. External validation and recalibration of the Brock model to predict probability of cancer in pulmonary nodules using NLST data. Thorax. 2019;74(6):551-63.

13. Ost DE, Gould MK. Decision making in patients with pulmonary nodules. Am J Respir Crit Care Med. 2012;185(4):363-72.

14. Adegunsoye A, Oldham JM, Bonham C, Hrusch C, Nolan P, Klejch W, et al. Prognosticating Outcomes in Interstitial Lung Disease by Mediastinal Lymph Node Assessment. An Observational Cohort Study with Independent Validation. Am J Respir Crit Care Med. 2019;199(6):747-59.

15. Ali N, Loughborough WW, Rodrigues JCL, Suntharalingam J, Hudson B, Hall T, et al. Computed tomographic and clinical features of pulmonary veno-occlusive disease: raising the radiologist's awareness. Clin Radiol. 2019;74(9):655-62.

16. Munden RF, Carter BW, Chiles C, MacMahon H, Black WC, Ko JP, et al. Managing Incidental Findings on Thoracic CT: Mediastinal and Cardiovascular Findings. A White Paper of the ACR Incidental Findings Committee. J Am Coll Radiol. 2018;15(8):1087-96.

17. Koda E, Yamashiro T, Onoe R, Handa H, Azagami S, Matsushita S, et al. CT texture analysis of mediastinal lymphadenopathy: Combining with US-based elastographic parameter and discrimination between sarcoidosis and lymph node metastasis from small cell lung cancer. PLoS One. 2020;15(12):e0243181.

18. Frank L, Quint LE. Chest CT incidentalomas: thyroid lesions, enlarged mediastinal lymph nodes, and lung nodules. Cancer Imaging. 2012;12(1):41-8.

19. Moons KG, Altman DG, Reitsma JB, Collins GS. Transparent Reporting of a Multivariate Prediction Model for Individual Prognosis or Development I. New Guideline for the Reporting of Studies Developing, Validating, or Updating a Multivariable Clinical Prediction Model: The TRIPOD Statement. Adv Anat Pathol. 2015;22(5):303-5.

20. Lin KF, Wu HF, Huang WC, Tang PL, Wu MT, Wu FZ. Propensity score analysis of lung cancer risk in a population with high prevalence of non-smoking related lung cancer. BMC Pulm Med. 2017;17(1):120.

21. Bae JM. Modifiable risk factors of lung cancer in "never-smoker" women. Epidemiology health. 2015;37:e2015047. 
22. Liang D, Wang J, Li D, Shi J, Jing J, Shan B, et al. Lung Cancer in Never-Smokers: A Multicenter Case-Control Study in North China. Front Oncol. 2019;9:1354.

23. Xu CC, Lei W, Jiang JH, Wang ZR, Ni CJ, Huang JA. Endobronchial ultrasound-guided transbronchial needle aspiration can improve the diagnostic accuracy of positron emission tomography/computed tomography in hilar and/or mediastinal lymphadenopathy. J Cancer Res Ther. 2019;15(7):1490-5.

24. Fournier C, Hermant C, Gounant V, Escarguel B, Thibout Y, Lachkar S, et al. Diagnostic of mediastinal lymphadenopathy in extrathoracic cancer: A place for EBUS-TBNA in real life practice? Respir Med Res. 2019;75:1-4.

25. Ten Haaf K, Jeon J, Tammemägi MC, Han SS, Kong CY, Plevritis SK, et al. Risk prediction models for selection of lung cancer screening candidates: A retrospective validation study. PLoS Med. 2017;14(4):e1002277.

26. Nemesure B, Clouston S, Albano D, Kuperberg S, Bilfinger TV. Will That Pulmonary Nodule Become Cancerous? A Risk Prediction Model for Incident Lung Cancer. Cancer Prev Res (Phila). 2019;12(7):463-70.

27. Tammemagi MC, Ten Haaf K, Toumazis I, Kong CY, Han SS, Jeon J, et al. Development and Validation of a Multivariable Lung Cancer Risk Prediction Model That Includes Low-Dose Computed Tomography Screening Results: A Secondary Analysis of Data From the National Lung Screening Trial. JAMA Netw Open. 2019;2(3):e190204.

28. Duan XQ, Wang XL, Zhang LF, Liu XZ, Zhang WW, Liu YH, et al. Establishment and validation of a prediction model for the probability of malignancy in solid solitary pulmonary nodules in northwest China. J Surg Oncol. 2021;123(4):1134-43.

29. Tang W, Peng Q, Lyu Y, Feng X, Li X, Wei L, et al. Risk prediction models for lung cancer: Perspectives and dissemination. Chin J Cancer Res. 2019;31(2):316-28.

30. González Maldonado S, Delorme S, Hüsing A, Motsch E, Kauczor H-U, Heussel C-P, et al. Evaluation of Prediction Models for Identifying Malignancy in Pulmonary Nodules Detected via Low-Dose Computed Tomography. JAMA Network Open. 2020;3(2):e1921221-e.

\section{Figures}

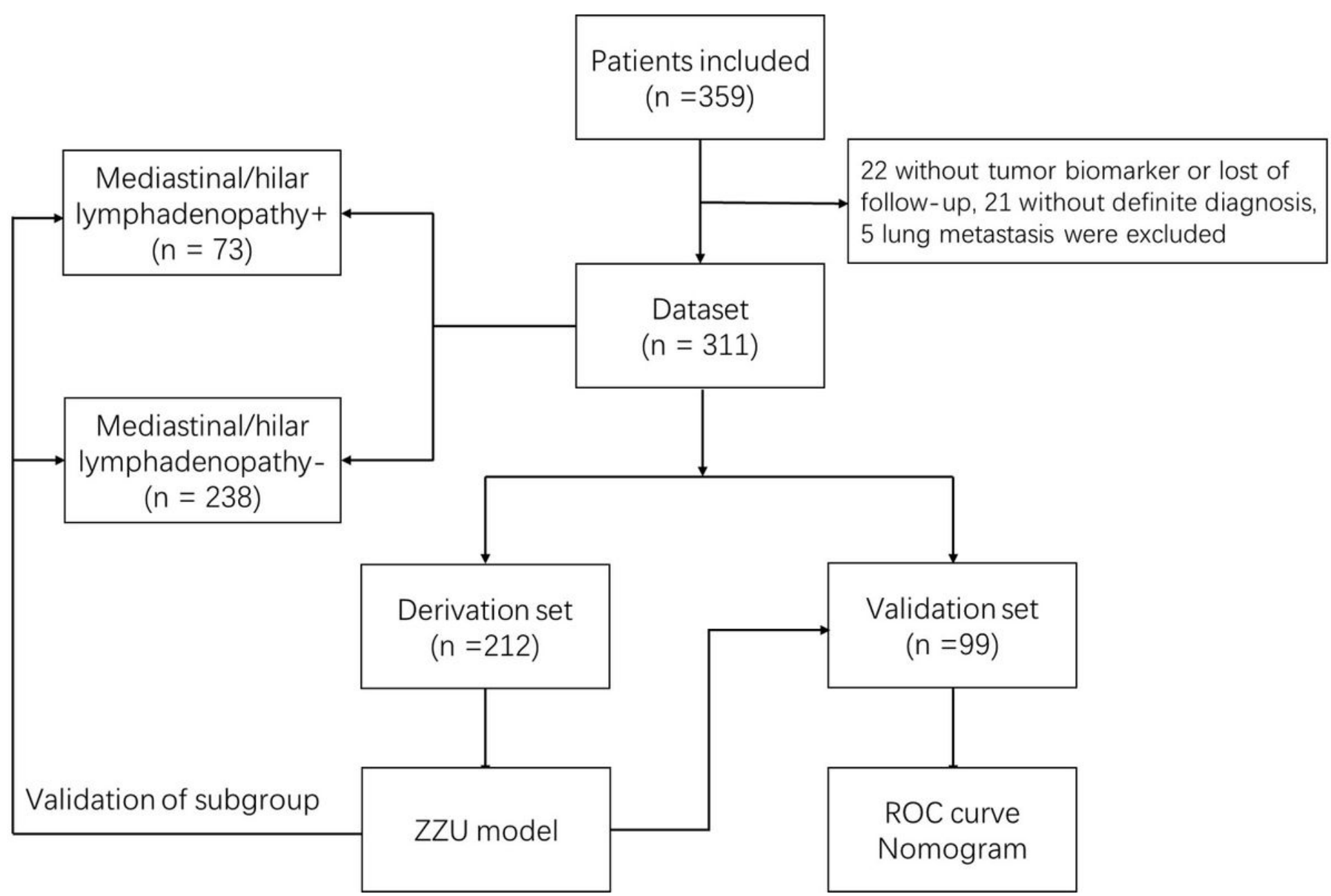

Figure 1

Page 10/13 


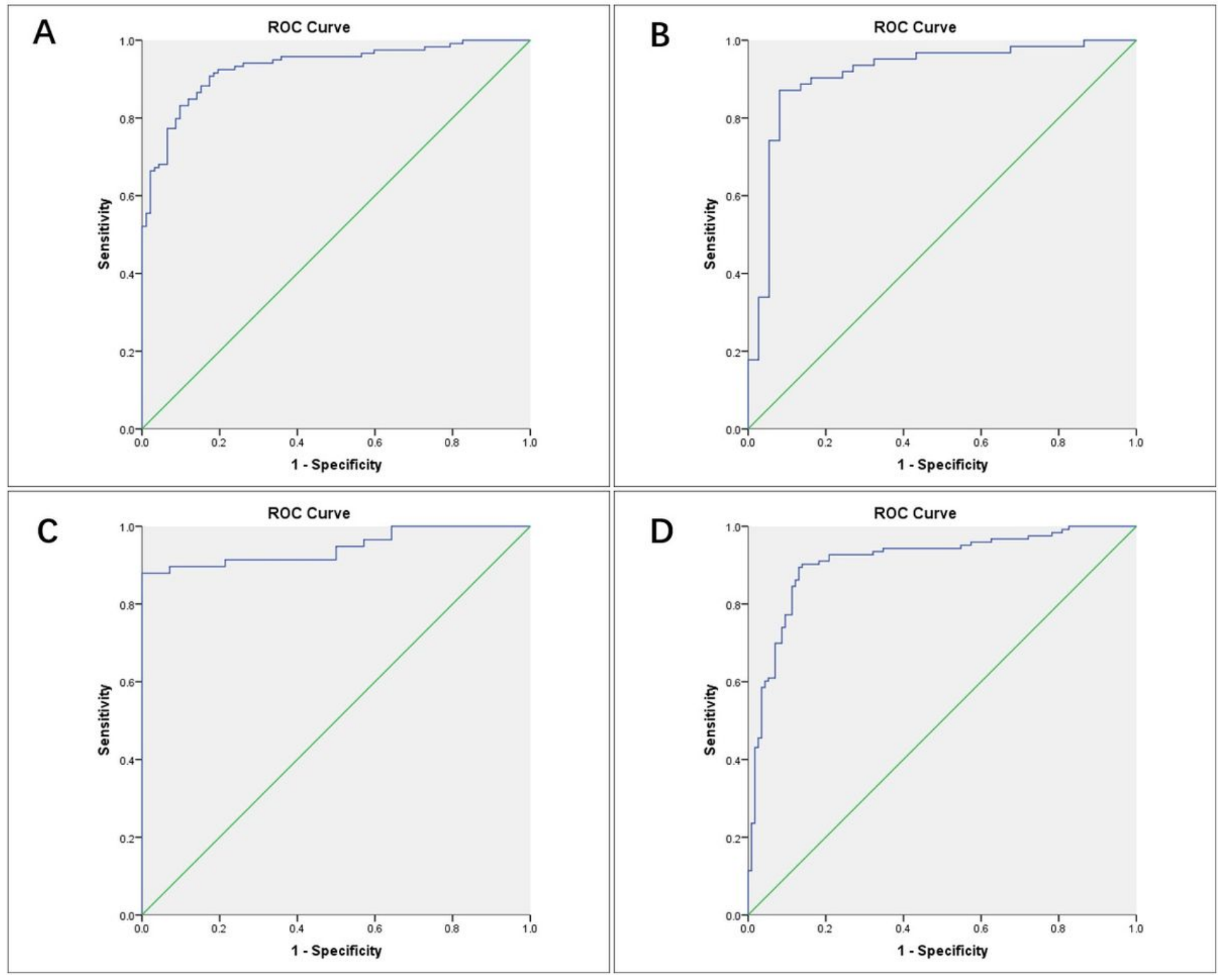

\section{Figure 2}

The ROC curve of the development and validation of the ZZU prediction model A. The ROC curve of the ZZU model in the training set (AUC $=0.93)$. B. The ROC curve of the ZZU model in the validation set $(A U C=0.91)$. C. The ROC of the validation of lung nodules with mediastinal/hilar lymphadenopathy $(A \cup C=0.95)$. D. The ROC of the validation of lung nodules without mediastinal/hilar lymphadenopathy $(A U C=0.91)$. ROC, receiver operating characteristic; $A U C$, area under the curve. 


\section{goodness of fit test}

30

25

20

15

10

5

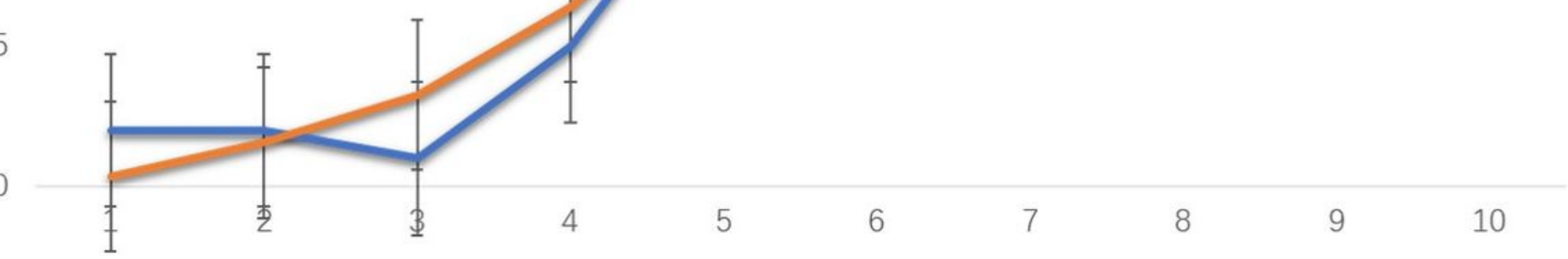

$-5$

$$
\text { -nodule }=1.00 \text { Observed } \quad \text { nodule }=1.00 \text { Expected }
$$

Figure 3

Goodness of fit test of the ZZU model 


Points
age
gender
number
sCA125
ML
lobulation
vascular_notch
sCYFRA211
Total Points
Risk of malignancy

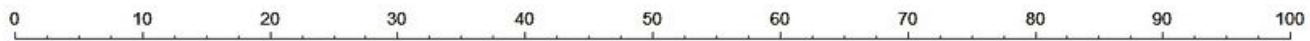
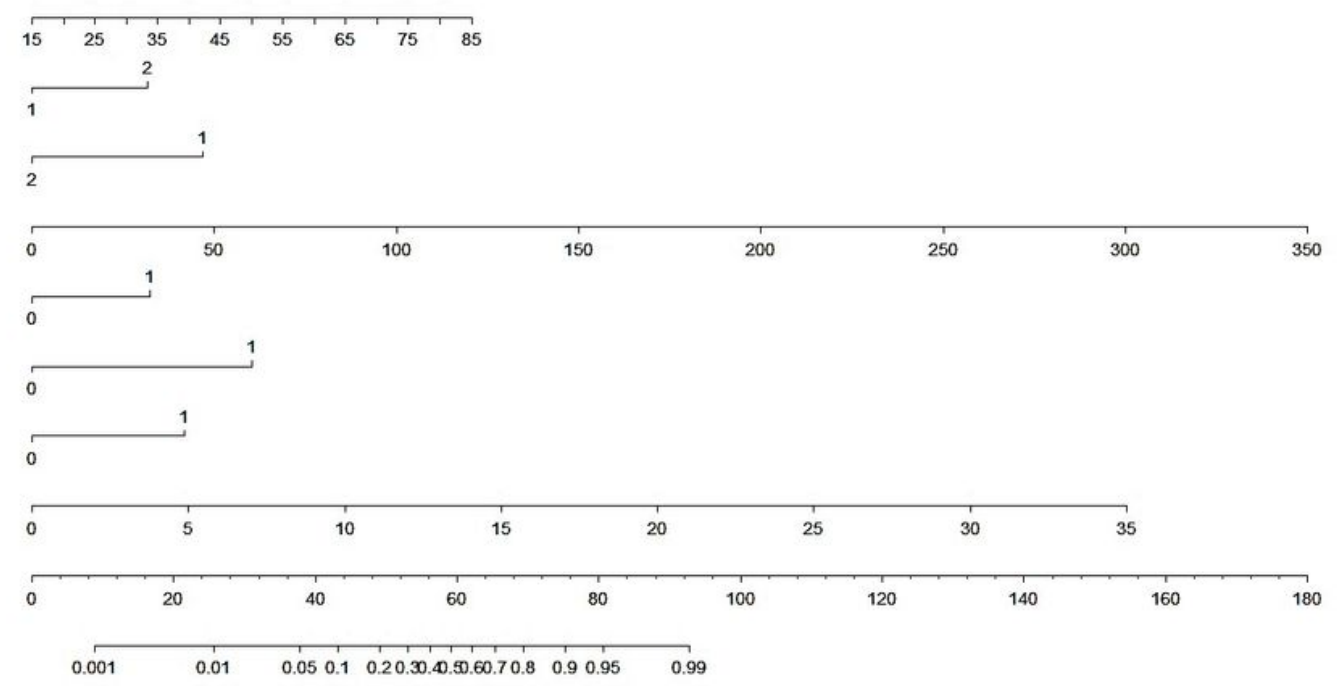

\section{Figure 4}

Nomogram of the prediction model for risk of malignancy of lung nodules Independent variables were visualized and the risk of malignancy calculated. For example, in a 65 year old patient, male, 2 lung nodules, CA125 $30 \mathrm{U} / \mathrm{ml}$, with mediastinal lymphadenopathy, lung nodule with lobulation, without vascular notch, CYFRA211 $5 \mathrm{U} / \mathrm{ml}$, the corresponding points scores are $25,0,0,8,10,17,0$ and 12 . The total points is 72 , and the risk of malignancy is 0.85 in the nomogram model. Abbreviations: ML, mediastinal/hilar lymphadenopathy. 